throughout the country. The project was the first of its kind, and the lessons learnt from it should allow subsequent surveys to achieve more with less effort. What procedure should be the next for a national audit of this kind? Tonsillectomy? Haemorrhoidectomy? Gastroscopy? Colectomy? There are many which deserve it as much if not more than laparoscopy.

\footnotetext{
${ }^{1}$ Gynaecological Laparoscopy. The Report of the Working Party of the Confidential Enquiry into Gynaecological Laparoscopy, eds G Chamberlain and J C Prown. London, Royal College of Obstetricians and Gynaecologists, 1978.

Phillips, J, et al, Fournal of Reproductive Medicine, 1976, 16, 105

Phillips, J, et al, Fournal of Reproductive Medicine, 1977, 18, 219.

${ }^{4}$ Phillips, J, et al, Fournal of Reproductive Medicine, 1977, 18, 227.
}

\section{A numbers game}

Necessarily, the value of laboratory tests (whether used for discriminating between health and disease, to aid diagnosis, or to monitor treatment) depends on the validity of the reported result. While it is generally accepted by both clinician and laboratory specialist that what is reported can only be an approximation to the truth, and that from time to time errors may occur, the process is given an air of respectability by the tacit acceptance of a normal or reference range of values. These ranges are statistically based and designed to minimise the overlap between values in health and disease. Morgan ${ }^{1}$ has recently reminded us that this naive approach can be misleading, for what we are doing is to compare a measurement at a single moment with a group of values. The central assumption is that the single value is truly representative of the person at that time.

The factors that influence a result are well recognised. Some affect specimen collection-venous stasis, haemolysis, and posture; among analytical factors are the method used and the standard of its performance; while age, sex, and race, and individual factors such as time of day, biological rhythms, diet, exercise, and drugs all contribute to the total variance. The larger the group, the more variables will be incorporated, the larger the reference range becomes, and the poorer will be its discrimination between health and disease. When these variables are reduced by selecting age- and sex-related ranges, attending to details of specimen collection, and using better analytical techniques, the ranges become more refined and apparently more useful. They ignore, however, the important component of intrapersonal variation.

Even if a relatively steady state is reached in an individual, then repeated samples still show a clear intrapersonal variation, ${ }^{2-4}$ and its degree differs for different constituents of biological fluids. ${ }^{45}$ Although intrapersonal and interpersonal variations are inextricably mixed, the greater the contribution of the former to total variance the smaller is the usefulness of the single-sample result. Morgan ${ }^{1}$ illustrates the practical problem from a study in Leeds in which two blood samples for potassium estimation were drawn from a group of patients with an interval of a month. Though the number of patients with hypokalaemia (11 and 15) was similar on the two occasions, only four had a low potassium concentration both times; the patients had a greater intrapersonal variation than did controls.

There is no simple solution. Clearly, every effort must be made to reduce as many of the known variables as possible, ${ }^{6}$ but paradoxically this increases the effect of intrapersonal variation. Replicate testing would give a better measure of an individual's "setting" for a given constituent, but that would raise laboratory and other costs. The ideal would be to test the individual against his own "normal" range-for departure from his own normal is more important than from the conventional group reference ranges. This means serial measurements over a period-one justification for the practice of some laboratories that indulge in cumulative reporting. ${ }^{7}$ Possibly statistical techniques would provide another alternative ${ }^{6}$; but for the present, just as the patient is the best specimen container, equally he seems to be the best guide to biochemical markers of his own health.

\footnotetext{
${ }^{1}$ Morgan, D B, Annals of Clinical Biochemistry, 1978, 15, 49

2 Williams, G Z, et al, Clinical Chemistry, 1970, 16, 1016

${ }^{3}$ Harris, E K, et al, Clinical Chemistry, 1970, 16, 1022.

${ }^{4}$ Pickup, J F, et al, Clinical Chemistry, 1977, 23, 842

' Harris, E K, Clinical Chemistry, 1974, 20, 1535.

${ }^{6}$ McPherson, K, et al, Clinical Chimica Acta, 1978, 84, 373.

${ }^{7}$ Harris, E K, Clinical Chemistry, 1976, 22, 1343.
}

\section{Serotonin, platelets, and autism}

In recent years there has been a spate of research into the role of serotonin (5-hydroxytryptamine, $5 \mathrm{HT}$ ) in neuropsychiatric conditions in childhood. ${ }^{12}$ Serotonin is concerned in synaptic transmission in the central nervous system, and abnormalities in blood serotonin concentrations have been reported in a wide range of neurological and psychiatric disorders. Close parallels exist between the properties of blood platelets and serotonergic synaptosomes in the central nervous system, and the platelet has come to serve as a model of a serotonergic neurone. ${ }^{2}$ But, while there is probably a common mechanism for serotonin binding in the two locations, platelets are more directly exposed to the environment than are brain cells, and the efflux of serotonin from platelets is not necessarily comparable to transmitter release at synapses. ${ }^{3}$ Nevertheless, such parallels have been suggested, and attention has been focused on the possibility of serotonin dysfunction in infantile autism. ${ }^{4}$

Schain and Freedman ${ }^{5}$ were the first to note high blood concentrations of $5 \mathrm{HT}$ in a proportion of children loosely diagnosed as autistic. Similar high concentrations were also found in non-autistic children with severe mental retardation. Since then better controlled investigations have confirmed both observations. ${ }^{6}$ Serotonin concentrations are raised in about a third of autistic children and about half of severely retarded children ${ }^{7}$ other than those with Down's syndrome (in whom the concentrations are abnormally low ${ }^{1}$ ), and in individual children they appear to be stable over several years.? The mechanisms remain obscure. The increase in 5HT does not appear to be due to diet, medication, or other extraneous influences, or to differences in the number of platelets or alterations in monoamine oxidase activity. ${ }^{7-9}$ Cohen et al ${ }^{10}$ have reported lower concentrations of the serotonin metabolite 5-hydroxyindolacetic acid (5HIAA) in the cerebrospinal fluid of autistic than in that of non-autistic psychotic children. The interpretation of that finding is far from clear, however, since neither group differed significantly from controls, whose 5HIAA concentrations were intermediate between the autistic and psychotic groups.

Another approach has been to study the uptake and efflux of serotonin from the platelets of autistic children. ${ }^{6}$ Boullin et $a l^{11}$ reported increased serotonin efflux as a finding specific to autism, but this was not confirmed by Yuwiler et al. ${ }^{12}$ Whether the conflicting results reflect differing biochemical 